\title{
Power Allocation and Mode Selection in Unmanned Aerial Vehicle Relay Based Wireless Networks
}

\author{
Qian Zeng ${ }^{1}$, Wei Huangfu ${ }^{1}$ and Tong Liu $^{2 *}$ \\ ${ }^{1}$ Beijing Engineering and Technology Center for Convergence Networks and Ubiquitous Services, \\ University of Science and Technology Beijing (USTB), \\ Beijing, China 100083 \\ ${ }^{2}$ The Department of Information and Communication Engineering, Harbin Engineering University, 145 Nantong \\ Street, Harbin, China 150001 \\ [e-mail: zengqian617@foxmail.com; huangfuwei@ustb.edu.cn; liutong@hrbeu.edu.cn] \\ *Corresponding author: Tong Liu
}

Received July 2, 2017; accepted November 3, 2018;

published February 28, 2019

\begin{abstract}
Many unmanned aerial vehicle (UAV) applications have been employed for performing data collection in facilitating tasks such as surveillance and monitoring objectives in remote and dangerous environments. In light of the fact that most of the existing UAV relaying applications operate in conventional half-duplex (HD) mode, a full-duplex (FD) based UAV relay aided wireless network is investigated, in which the UAV relay helps forwarding information from the source $(S)$ node to the destination $(D)$. Since the activated UAV relays are always floating and flying in the air, its channel state information (CSI) as well as channel capacity is a time-variant parameter. Considering decode-and-forward (DF) relaying protocol in UAV relays, the cooperative relaying channel capacity is constrained by the relatively weaker one (i.e. in terms of signal-to-noise ratio (SNR) or signal-to-interference-plus-noise ratio (SINR)) between $S$-to-relay and relay-to- $D$ links. The channel capacity can be optimized by adaptively optimizing the transmit power of $S$ and/or UAV relay. Furthermore, a hybrid $\mathrm{HD} / \mathrm{FD}$ mode is enabled in the proposed UAV relays for adaptively optimizing the channel utilization subject to the instantaneous CSI and/or remaining self-interference (SI) levels. Numerical results show that the channel capacity of the proposed UAV relay aided wireless networks can be maximized by adaptively responding to the influence of various real-time factors.
\end{abstract}

Keywords: Unmanned Aerial Vehicle, Full-Duplex, Hybrid Mode Selection, Channel Capacity, Power Allocation.

This work was supported by the key project of the National Natural Science Foundation of China (No. 61431001), the open research fund of National Mobile Communications Research Laboratory Southeast University (No.2017D02), Key Laboratory of Cognitive Radio and Information Processing, Ministry of Education (Guilin University of Electronic Technology), and the Foundation of Beijing Engineering and Technology Center for Convergence Networks and Ubiquitous Services. 


\section{Introduction}

Unmanned aerial vehicles (UAVs), as a critical part of the unmanned aircraft systems that controlled by the ground control stations [1], may act as relays for enabling the constitution of a contemporary self-organized network [2][3] infrastructure that applied in some emergence scenarios such as hazard and/or dangerous rescue area. By establishing a direct communication link between the UAVs and the ground-based vehicle stations [4], a robust communication can be enabled in modern battlefield scenarios [5].

Many studies have been focused on the implementations of UAV based relays ${ }^{1}$. For instance, the authors in [10] designed a relay trajectory together with optimal source/relay transmit power adaption for maximizing the sum throughput. When it comes to multi-ground units, the authors in [11] investigated the application of UAVs by identifying the optimal UAV positions without necessarily knowing the ground units' positions. For multiple-UAV scenarios, the trajectories between neighboring UAVs must be considered, as investigated by [7], in which the authors proposed an effective dynamic trajectory control algorithm for improving the communication performance in terms of delay and throughput by adapting the distance between the centers of trajectories of two neighboring UAVs.

Despite all this, there still exist several challenges in implementing UAV-based networks. Since the employment of multiple UAVs can substantially extend the communication range [13], multiple-input-multiple-output (MIMO) based UAVs can be utilized for further improving the channel capacity [9], however, with a higher computational/control complexity required. Furthermore, the limited battery power supply also constitutes a big challenge to the practical application of UAVs. To address the above-mentioned issue, the authors in [6] proposed an optimal scheduling scheme to guarantee a success rate as well as balance the energy consumption in a swarm of UAVs. In addition, since the exiting Medium Access Control (MAC) protocols are inappropriate to implement in UAV-based networks, it highly requires a novel MAC protocol to be proposed for facilitating a high throughput, user fairness and spectrum/power efficiency in densely deployed UAV networks [14].

In light of the fact that the full-duplex (FD) mode relays [15] can be implemented in the UAVs for further improving the spectrum utilization, several studies have been carried out in investigating the potential benefits offered by employing FD techniques [16]. In theory, FD mode is capable of doubling the throughput over the conventional half-duplex (HD) mode [17], provided that the self-interference (SI) ${ }^{2}$ imposed on the FD devices can be sufficiently suppressed. In particular, hybrid HD/FD relay mode may be an appropriate choice for optimizing the sum throughput in practical systems [18].

Unlike the existing relay selection techniques that usually require multiple candidate relays [19], the hybrid mode only needs one relay, which can adaptively adjust its transmission mode according to the instantaneous CSI [20]. In [21], the authors classified the FD relay system

\footnotetext{
${ }^{1}$ Basically, we may create a cooperative communication system by employing various types of UAVs, such as fix wing [1], [6]-[10] and rotate wing [11], [12] UAVs. Furthermore, relays installed in balloons may also offer a promising solution for cooperative relaying networks. For example, the high altitude balloon UAVs can be employed for facilitating high speed Internet access [8].

${ }^{2}$ In FD mode, the SI mainly comes from the large power difference between the FD devices' own transmission and the useful signal from the remote transmitters. If the SI power cannot be sufficiently suppressed, the performance of FD systems may even be worse than that of the convention HD mode [15]. As the SI is fluctuant with the system power allocation, capacity of FD systems may not always be higher than that of the HD systems. Since SI cancellation is beyond the scope of this paper, the interested readers may refer to [15] for details.
} 
into four categories, including i)the scenario with a single FD relay and two HD source nodes [18], ii) the one with a single FD relay and two FD source nodes [22], iii) the one with a single FD relay/source node and a single HD source node [23], and iv) the one with a single HD relay with two source nodes, in which at least one source node working in FD mode [21]. Note that the FD relays may suffer from not only residual SI but also inter-relay interference [24]. In UAV aided communications, FD and/or hybrid mode may offer a suitable way for relieving the capacity limit of the existing HD mode UAV aided systems [6]. So far, hybrid HD/FD mode selection techniques have been investigated in several scenarios, including multi-input multi-output (MIMO) [25], heterogeneous network [26], femtocell network [27], security-ganrantee systems [28], buffer aided systems [29], and energy harvesting systems [30], etc.

However, a new challenge may be imposed on UAV aided systems, because the UAV relays are always floating and flying, resulting in a dynamic receive signal at the receiver. Meanwhile, the capacity of FD mode UAV relays may be limited by the residual SI power. In light of the fact that the hybrid mode selection may be the best choose for UAV aided systems, in this paper, we will investigate the mode selection strategies in UAV aided relay subject to both SI cancellation and adaptive power allocation.

The remainder of this paper is organized as follows: after the introduction in Section 1, the system model is provided in Section 2, followed by describing the problem of formation in Section 3. In Section 4, we analysis the performance of the proposed UAV aided systems. Finally, Section 5 concludes this paper.

\section{System and Channel Models}

The proposed system model will be given out in this section, followed by introducing the related channel model as well as its capacity.

\subsection{System Mode}

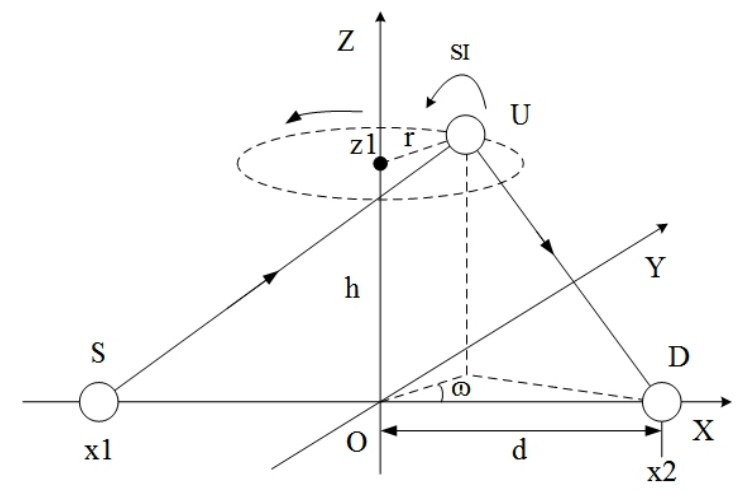

Fig. 1. Geometry model of the proposed UAV aided systems.

The proposed system model is illustrated in Fig. 1, the main symbols notation is shown in Table 1. In Fig. 1, three nodes are comprised: Node $S$ denotes the source node, node $D$ is the destination node, and node U stands for a fix-wing UAV. The distance between S and D is time variant. Furthermore, the UAV hovers over $S$ and $D$ and forwards signal from the former to the latter. Without loss of generality, both S and D are assumed to work in HD mode, but UAV can switch its working mode between FD and HD modes. When the UAV works in FD mode, an appropriate SI cancellation capability should be enabled in it. 
Table 1. Main symbols notation

\begin{tabular}{|c|c|}
\hline Symbol & Notation \\
\hline \hline Node S/S & The source node \\
\hline Node D/D & The destination node \\
\hline$d, h, r$ & The fix-wing UAV \\
\hline$\omega$ & The distance length in the model \\
\hline$d_{a, b}, a, b \in\{\mathrm{S}, \mathrm{D}, \mathrm{U}\}$ & The angular velocity \\
\hline$H_{a, b}, \sigma_{a, b}^{2} a, b \in\{\mathrm{S}, \mathrm{D}, \mathrm{U}, \mathrm{I}\}$ & The distance between two nodes \\
\hline$\alpha_{a, b}, a, b \in\{\mathrm{S}, \mathrm{D}, \mathrm{U}, \mathrm{I}\}$ & The channel coefficients and its variance \\
\hline$\gamma_{a, b}, a, b \in\{\mathrm{S}, \mathrm{D}, \mathrm{U}, \mathrm{I}\}$ & The channel path loss \\
\hline$X_{a}, a, b \in\{\mathrm{S}, \mathrm{U}\}$ & The transmitted information \\
\hline$P_{a}, a, b \in\{\mathrm{S}, \mathrm{U}\}$ & The maximum transmission power \\
\hline$C_{H D}, C_{F D}, C_{D T}$ & The channel capacity of HD, FD, DT mode \\
\hline
\end{tabular}

In the three-dimensional Cartesian coordinate systems, the location of $S$ can be represented as $(x 1,0,0)$, where $x 1=d$. Similarly, the location of $D$ can be given by $(x 2,0,0)$, where $x 2=-d$. Furthermore, UAV takes location $(0,0, z 1)$, where $z 1=h$, which is regarded as the center for circling in constant speed $\omega$. In addition, the turning radius is assumed to be $r$, making the location of UAV be

$$
L_{U}(r, \omega, h)=(r \cos \omega, r \sin \omega, h)
$$

Thus, the distance between each pair of nodes is given by

and

$$
\begin{aligned}
& d_{S U}=\left(h^{2}+r^{2}+d^{2}-2 r d \cos \omega\right)^{\frac{1}{2}} \\
& d_{U D}=\left(h^{2}+r^{2}+d^{2}+2 r d \cos \omega\right)^{\frac{1}{2}}
\end{aligned}
$$

$$
d_{S D}=2 d
$$

where $d_{S U}, d_{S D}$ and $d_{U D}$ denote the $S$-to-UAV, UAV-to-D and $S$-to- $D$ distances, respectively.

\subsection{Channel Model}

For simplicity, we assume that the transmission channel suffer from large-scale path loss, and statistically independent small-scale quasi-static frequency nonselective Rayleigh fading. Consequently, the complex channel coefficients $H_{S U}, H_{U D}, H_{S D}$ are assumed to be independently and circularly symmetric complex Gaussian random variables with zero mean and unit variance, i.e. $H_{S U} \sim C N\left(0, \sigma_{S U}^{2}\right), H_{U D} \sim C N\left(0, \sigma_{U D}^{2}\right), H_{S D} \sim C N\left(0, \sigma_{S D}^{2}\right)$, $\sigma_{S U}{ }^{2}=\sigma_{U D}{ }^{2}=\sigma_{S D}{ }^{2}=1$, and the normalized unit power can be given by $E\left\{\left|H_{S U}\right|^{2}\right\}=E\left\{\left|H_{U D}\right|^{2}\right\}=E\left\{\left|H_{S D}\right|^{2}\right\}=1$. Note that the above-mentioned parameters are assumed to be known to both the receiver side and the transmitter side. Furthermore, a perfect timing and frequency synchronization is assumed to be available in the proposed system. $H_{S I}$ is the self-interference channel which can be estimated [15]. In addition, we assume that the transmitted information for $S$ and $U$ (i.e. $X_{S}$ and $X_{U}$, respectively) follows a circularly symmetric complex Gaussian distribution $C N(0,1)$, whose expectation is given by $E\left\{\left|X_{S}\right|^{2}\right\}=E\left\{\left|X_{U}\right|^{2}\right\}=1$. 
The receive signal at UAV in FD and HD mode are respectively given by

$$
Y_{U_{-} F D}=H_{S U} \sqrt{a_{S U}} \sqrt{P_{S}} X_{A}+H_{S I} \sqrt{a_{S I}} \sqrt{P_{U}} X_{U}+N_{U}
$$

and

$$
Y_{U_{-} H D}=H_{S U} \sqrt{a_{S U}} \sqrt{P_{S}} X_{A}+N_{U}
$$

respectively, where $a_{S U}$ is the channel path loss of between $S$ and UAV, $P_{S}$ and $P_{U}$ denote the transmission power of S and UAV, respectively, and $a_{S I}$ stands for the SI channel attenuation factor in UAV. We denote the SI cancellation capability of UAV by $\sqrt{\tilde{a}_{S I}}$, and we may simply use $H_{S I} \sqrt{a_{S I}}=H_{S I} \sqrt{\tilde{a}_{S I}}$ in the following. Furthermore, $N_{U}$ is the additive white Gaussian noise (AWGN) in UAV with distribution $C N\left(0, P_{N, U}\right)$, where $P_{N, U}$ denotes the variance of noise.

The receive signal at $D$ in FD, $\mathrm{HD}$ and DT mode are respectively given by

$$
\begin{gathered}
Y_{D_{-} F D}=H_{U D} \sqrt{a_{U D}} \sqrt{P_{U}} X_{U}+H_{S D} \sqrt{a_{S D}} \sqrt{P_{S}} X_{S}+N_{D} \\
Y_{D_{-} H D}=H_{U D} \sqrt{a_{U D}} \sqrt{P_{U}} X_{U}+N_{D}
\end{gathered}
$$

and

$$
Y_{D_{-} D T}=H_{S D} \sqrt{a_{S D}} \sqrt{P_{S}} X_{S}+N_{D}
$$

The attenuation factor associated with path loss are respectively given by $a_{S U}=K_{S U} / d_{S U}^{\alpha}$, $a_{U D}=K_{U D} / d_{U D}^{\alpha}$ and $a_{S D}=K_{S D} / d_{S D}^{\alpha}, K_{S U}, K_{U D}$ and $K_{S D}$ are environment specified constraint conditions, and $\alpha$ is the path loss exponent, $K_{S U}=K_{U D}=K_{S D}=K=G_{T} G_{R} \lambda^{2} /(4 \pi)^{2}$, where $G_{T}$ and $G_{R}$ denote antenna gains at transmitter and receiver, and $\lambda$ is the wavelength.

\subsection{The SINRs and Capacity}

We use the normalized channel signal-to-noise ratios (SNRs) to parameterize the system as $\gamma_{S U}=\left(\left|H_{S U}\right|^{2} a_{S U}\right) / P_{N, U} \quad, \quad \gamma_{U D}=\left(\left|H_{U D}\right|^{2} a_{U D}\right) / P_{N, D} \quad, \quad \gamma_{S D}=\left(\left|H_{S D}\right|^{2} a_{S D}\right) / P_{N, D} \quad$ and $\gamma_{S I}=\left(\left|H_{S I}\right|^{2} a_{S I}\right) / P_{N, U}$ with the particular setting of maximum transmission power usage $\left(P_{S}=P_{U}=1\right)[18]$.

In FD mode: the received signals are given by (5) and (7), leading to the SINRs in UAV and $D$ as [18]: $\gamma_{U}=\left(P_{S} \gamma_{S U}\right) /\left(P_{U} \gamma_{S I}+1\right)$ and $\gamma_{D}=\left(P_{U} \gamma_{U D}\right) /\left(P_{S} \gamma_{S D}+1\right)$, respectively.

The end-to-end SINR of FD mode is thus given by

$$
\gamma_{F D}=\min \left\{\gamma_{U}, \gamma_{D}\right\}
$$

Accordingly, the channel capacity of FD mode is

$$
C_{F D}=\log _{2}\left(1+\gamma_{F D}\right)
$$

In HD mode: Using maximum ratio combing (MRC) [31], the end-to-end SNR is given by

Without MRC, we have $\gamma_{S D}=0$

$$
\gamma_{H D}=\min \left\{P_{S} \gamma_{S U}, P_{U} \gamma_{R D}+P_{S} \gamma_{S D}\right\}
$$

Similarly, the channel capacity of HD mode is given by

$$
C_{H D}=0.5 \log _{2}\left(1+\gamma_{H D}\right)
$$

In DT mode: the source $S$ uses the maximum transmission power to achieve the optimal SNR, which obviously leads to 


$$
\gamma_{D T}=P_{S} \gamma_{S D}
$$

The channel capacity of DT mode is thus given by

$$
C_{D T}=\log _{2}\left(1+\gamma_{D T}\right)
$$

\section{Problem Formulation}

As the UAV relay is floating over the air, the distance between UAV and $S$ and that between UAV and $D$ are always changing, thus making both $\gamma_{U}$ and $\gamma_{D}$ be time variant. From ((11) and (13), the capacity of $C_{H D}$ and $C_{F D}$ will be different. Meanwhile, the SI cancellation ability also constrains the capacity of $C_{F D}$. As we know, $C_{H D}$ may be higher than $C_{F D}$ under some transmission environment. We will analyze the best capacity transmission strategy under variant scenarios. First, let us define the total energy of $S$ and UAV in each transmission cycle $T$ subject to two constraints:

Total power constraint: When the total transmit power of $S$ and UAV are limited, their transmission power will be constrained by each other, namely $P_{S}+P_{U} \leq 1$ in FD and HD modes, and $P_{S}=1$ and $P_{U}=0$ in DT mode.

Independent power constraint: The transmit power at $\mathrm{UAV}$ and $R$ are restricted by $P_{S} \leq 1$ and $P_{U} \leq 1$, respectively. In DT mode, on the other hand, $P_{S}$ always equals 1 , and $P_{U}=0$ always satisfies.

In FD mode, since $S$ and UAV transmit simultaneously, UAV may suffer SI from its own transmission, while $D$ will receive interference from $S$ when it is listening to UAV. From (10), to achieve $\gamma_{U}=\gamma_{D}$, the optimal $P_{S}$ and $P_{U}$ may need a proper adjustment. From (12), the optimized power is achieved by $P_{S} \gamma_{S U}=P_{U} \gamma_{R D}+P_{S} \gamma_{S D}$, where the optimal $P_{S}$ and $P_{U}$ in HD also require a proper adjustment.

\subsection{Transmit Power Adaption}

From (10), in FD transmission mode, when $P_{S}$ is known, the optimal $P_{U}$ can be obtained through $\gamma_{U}=\gamma_{D}$, leading to

$$
P_{U_{-} F D}^{*}=\min \left\{P_{U_{-} F D M A X} \quad, \frac{1}{\gamma_{S I}}\left(\sqrt{\frac{P_{S} \gamma_{S I} \gamma_{S U}\left(P_{S} \gamma_{S D}+1\right)}{\gamma_{U D}}+\frac{1}{4}}-\frac{1}{2}\right)\right\}
$$

In total-power-constraint mode, we have $P_{U_{-} F D M A X}=1-P_{S}$. In independent power constraint mode, on the other hand, we have $P_{U_{-} \text {FDMAX }}=1$.

Similarly, in HD mode, from (12), we can obtain

$$
P_{U_{-} H D}^{*}=\min \left\{P_{U_{-} H D M A X} \quad, \frac{P_{S}\left(\gamma_{S U}-\gamma_{S D}\right)}{\gamma_{U D}}\right\}
$$

In total-power-constraint mode, we get $P_{U_{-} \text {HDMAX }}=1-P_{S}$. In independent power constraint mode, on the other hand, we get $P_{U_{-} \text {HDMAX }}=1$. Furthermore, without implementing MRC, we have $\gamma_{S D}=0$.

From (16), it is shown that $P_{U}$ is determined by a variety of parameters, including $\gamma_{S I}, P_{S}$, $\gamma_{S U}, \gamma_{S D}$ and $\gamma_{U D}$. We may rewrite it as 


$$
\gamma_{S I} \leq \frac{P_{S} \gamma_{S U}\left(P_{S} \gamma_{S D}+1\right)}{\gamma_{U D} P_{U_{-} F D M A X}}-\frac{1}{P_{U_{-} F D M A X}}
$$

When the result on the right side of the above-mentioned equation is greater than that of the left side, the system should carry out power limitation operation. When the system's SI cancellation capability is fixed, using a higher $P_{S}$ may not always lead to a better performance. In this case, whether the system requires power limitation is related to the UAV's location.

From (17), power limitation is required if

$$
P_{U_{-} H D M A X} \leq \frac{P_{S}\left(\gamma_{S U}-\gamma_{S D}\right)}{\gamma_{U D}}
$$

is satisfied.

\subsection{Relaying Modes Selection Analysis}

When we take both (16) and (10) into ((11), and take both (17) and (12) into (13), we will get the corresponding system capacity. Note that the capacity in each transmission mode is determined by several variables corresponding to the above-mentioned statement. We can also rewrite the formula ((11), (13) and (15) as

$$
\begin{gathered}
C_{F D}=f_{F D}\left(\gamma_{S I}, P_{S}, L_{U}\right) \\
C_{H D}=f_{H D}\left(P_{S}, L_{U}\right)
\end{gathered}
$$

and

$$
C_{D T}=f_{D T}\left(P_{S}, d\right)
$$

respectively. To optimize the end-to-end performance, the UAV will carry out hybrid relay selection to switch to a proper mode according its instantaneous CSI. The ultima spectral efficiency is thus given by

$$
C_{\text {hybrid }}= \begin{cases}\max \left\{C_{F D}, C_{H D}, C_{D T}\right\} & , \gamma_{S U} \neq 0 \\ \max \left\{C_{F D}, C_{H D}\right\} & , \gamma_{S U}=0\end{cases}
$$

\section{Performance Analysis}

In practical scenarios, there may exist some complex terrain conditions, making the source node and destination node unable to communication directly. In the following, we first analyze the transmission condition in which the DT mode exists, followed by analyzing the condition without the DT mode. After that, we focus on the hybrid relay selection without considering the DT mode.

\subsection{When to Use DT Mode}

In this case, we assume that the SI can be totally cancelled. The critical parameters impacting the performance of the proposed system will be $K=(1 * 1 * 1 / 3) /(4 * \pi)^{2}, h=10, r=40$, $a=3, d \in(20,50)$.

In total-power-constraint mode, $S$ 's transmission power may take $P_{S}=0.1,0.5,0.9$, because both $S$ and UAV will have no power supply when $P_{S}=0$ or $P_{S}=1$. We can analyze the capacity in terms of both the UAV's location and $D$. Numerical results are shown in Fig. 2Fig. 6. 


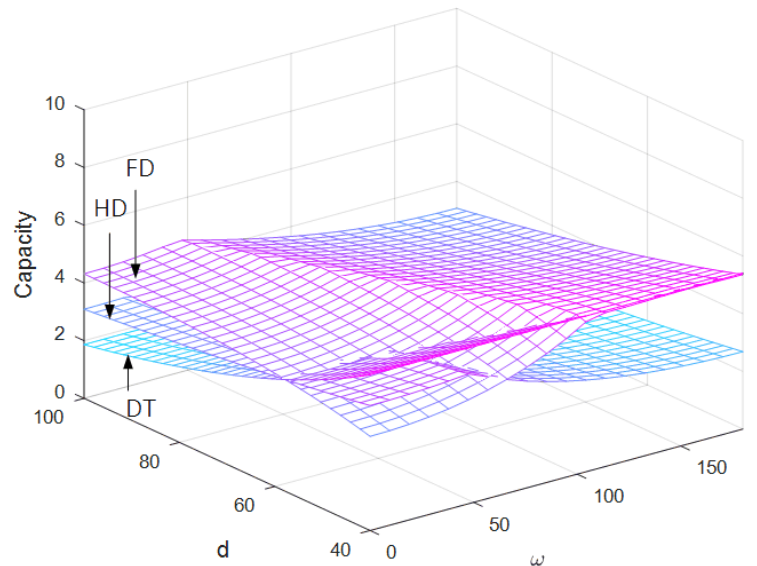

Fig. 2. Capacity with $P_{S}=0.1$ in total-power-constraint mode.

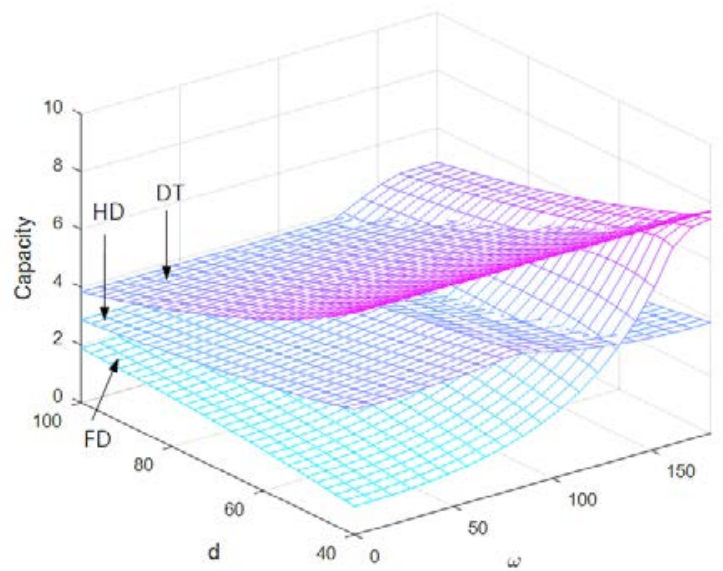

Fig. 3. Capacity with $P_{S}=0.5$ in total-power-constraint mode.

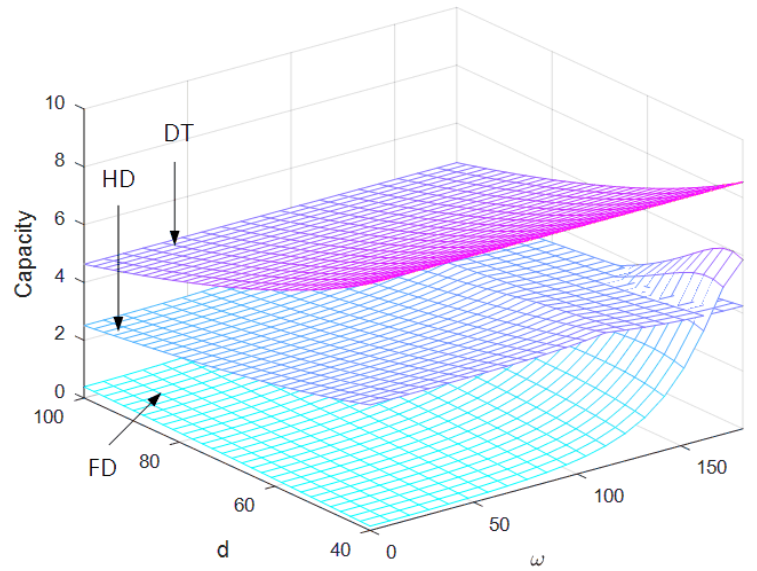

Fig. 4. Capacity with $P_{S}=0.9$ in total-power-constraint mode. 
1) From the first three figures, the superposition of three curved surfaces changes when $P_{S}$ becomes larger, with the gap between different capacity values increased gradually.

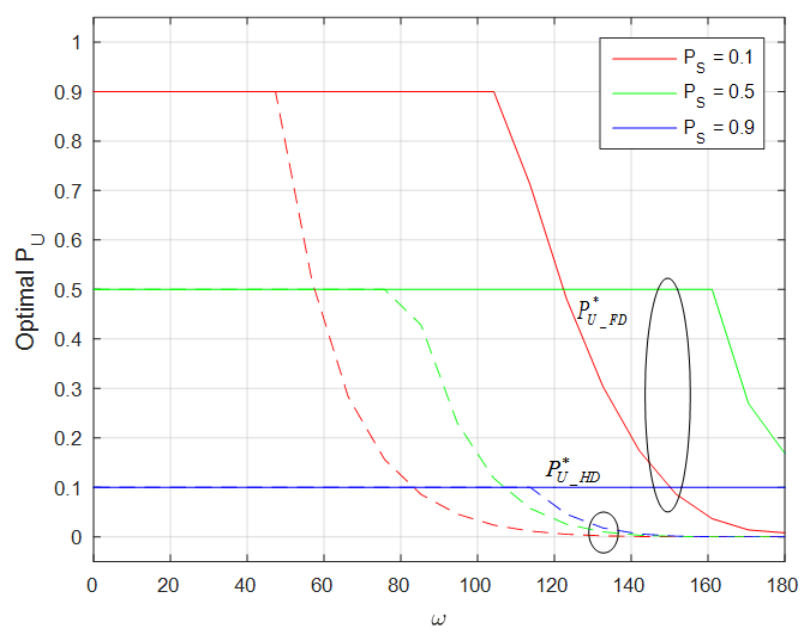

Fig. 5. Optimal $P_{U}$ under variant $\omega$ in total-power-constraint mode.

- When $P_{S}=0.1$, the capacity of FD mode generally outperforms that of HD mode. However, the capacity in DT mode may be better than the other two modes when $S$ and $D$ are close to each other. Evidently, the lower $P_{S}$ leads to a better performance in FD mode.

- When $P_{S}=0.5$, the attainable capacity in HD mode is basically remains unchanged (i.e. about $2.5 d B$, which is less than that in DT mode). When UAV moves from $S$ to $D$, the capacity of FD mode is drastically increased. Furthermore, when $\omega$ approaches $130^{\circ}$, the capacity in FD mode becomes higher than that in DT mode, implying that increasing $P_{S}$ from 0.1 to 0.5 makes the capacity of DT significantly be increased. However, the capacity in FD mode exhibits a great fluctuation.

- When $P_{S}=0.9$, the capacity in DT mode outperforms that of both FD and HD modes (the capacity in HD mode remains at 2.5dB). Unfortunately, the capacity in FD mode is almost lower than HD mode at $0 d B$. Only in case that $S$ is closed to UAV and UAV is closed to $D$, the capacity in FD mode becomes better. The highest $P_{S}$ results in the lowest capacity in FD mode.

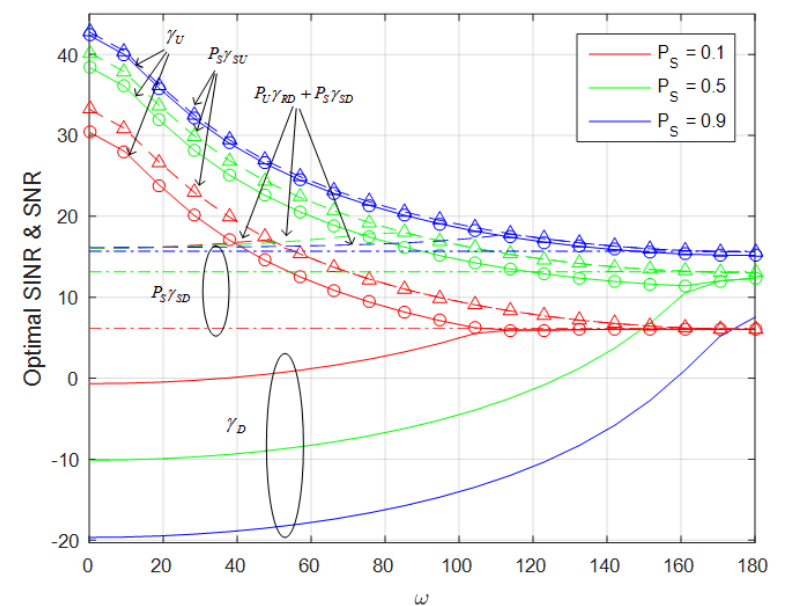

Fig. 6. Optimal SNRs and SINRs under variant $\omega$ in total-power-constraint mode. 
From (22), the capacity in DT mode, which is a function of $D$, increases linearly with the increases of $P_{S}$. Therefore, the curved surface of capacity keeps invariant and shifts up with the increases of $P_{S .}$, as shown in Fig. 2, Fig. 3 and Fig. 4. However, from (20) and (21), the capacities in $\mathrm{HD}$ and FD modes are impacted by parameters such as $D, \omega$ and $P_{U_{-} \text {MAX }}$ simultaneously, that why the curved surfaces of $C_{F D}$ and $C_{H D}$ are shown to fluctuate and with the maximum value being attainable.

2) Let us display the optimal $P_{U}$ variation curves according to (16) and (17), as shown in Fig. 5. After that, we will display the SNRs and SINRs relationship between $S$-to-UAV and UAV-to- $D$ links as well as the SNRs between $S$ and $D$, as shown in Fig. 6. Without loss of generality, we define $d=40$.

- $\quad$ Fig. 5 shows the changes of $P_{U}$ in both FD and HD mode. When $P_{S}+P_{U} \leq 1$ and if the ideal optimal $P_{U}$ exceeds $1-P_{S}, P_{U}^{*}$ is being truncated (i.e. the value will be only equal to $1-P_{S}$ ), the curves keep horizontal. It is shown that the two curves associated with FD and HD modes coincide when the $P_{S}$ is fixed. When UAV is closed to $D$ (i.e. $\omega$ is increasing), $P_{U}^{*}$ is gradually decreased. When it is lower than $1-P_{S}$, it will begin turning into a concave downward curve for a certain $\omega$. Note that the above-mentioned case happens much more fast in HD mode than in FD mode.

- $\quad$ Fig. 6 shows the SINRs and SNRs variations of each links in FD, HD and DT modes. The downward trend curves in upper half of the figure describe the actual SINRs and SNRs on UAV that are working in FD or HD mode, namely $\gamma_{U}$ and $P_{S} \gamma_{S U}$. The other part of the curves denote the actual received SINRs and SNRs on $D$, as denoted by $\gamma_{D}$ and $P_{U} \gamma_{R D}+P_{S} \gamma_{S D}$. Furthermore, the horizontal lines in the middle represent SNRs of $P_{S} \gamma_{S D}$ in DT mode. In addition, $\gamma_{D}$ and $P_{U} \gamma_{R D}+P_{S} \gamma_{S D}$ coincide with $\gamma_{U}$ and $P_{S} \gamma_{S U}$ when $P_{U}=P_{U}^{*}$, and superposition with $P_{S} \gamma_{S D}$. According to (10) and (12), the capacity in FD and HD modes are determined by the minimum SINRs or SNRs of UAV and $D$, implying that the capacity of the system is determined by $\gamma_{D}$ and $P_{U} \gamma_{R D}+P_{S} \gamma_{S D}$. Compared to the turning point in Fig. 5, it is the same turning point in Fig. 6 where parameters $\gamma_{D}$ and $\gamma_{U}$, $P_{U} \gamma_{R D}+P_{S} \gamma_{S D}$ and $P_{S} \gamma_{S U}$ start to coincide. When the ideal $P_{U} \geq 1-P_{S}$ attainable, then $P_{U}^{*}=1-P_{S}$ is satisfied, implying that $\gamma_{U} \neq \gamma_{D}$ and $P_{S} \gamma_{S U} \neq P_{U} \gamma_{R D}+P_{S} \gamma_{S D}$. When UAV gradually approaches $D, \gamma_{U}$ and $P_{S} \gamma_{S U}$ subsequently decrease until $P_{U}^{*} \leq 1-P_{S}$, with $\gamma_{U}=\gamma_{D}, P_{S} \gamma_{S U}=P_{U} \gamma_{R D}+P_{S} \gamma_{S D}$ satisfied, as shown in Fig. 2, Fig. 3 and Fig. 4. with $d=40$.

In independent power constraint mode, since the upper limit of both $P_{S}$ and $P_{U}$ equals 1 , we may set $P_{S}$ to be $0.1,0.5$ or 1 . The simulation results are shown in Fig. 7, Fig. 8 and Fig. 9, the analysis are shown in Fig. 10 and Fig. 11.

1) There exists a relatively small difference with total power constraint mode when $P_{S}=0.1$, as shown in Fig. 2 and Fig. 7. When the transmission power of $S$ is small, the growth of the transmission power upper limit of $P_{U}$ has little impact on the overall capacity. When $P_{S}=0.5$, as shown in Fig. 8, the capacity in TD mode is the same as that in Fig. 3, while the capacities of FD and HD modes become a litter higher (but the relationship between the three curved surfaces dies not change too much), implying that the independent power constraint 


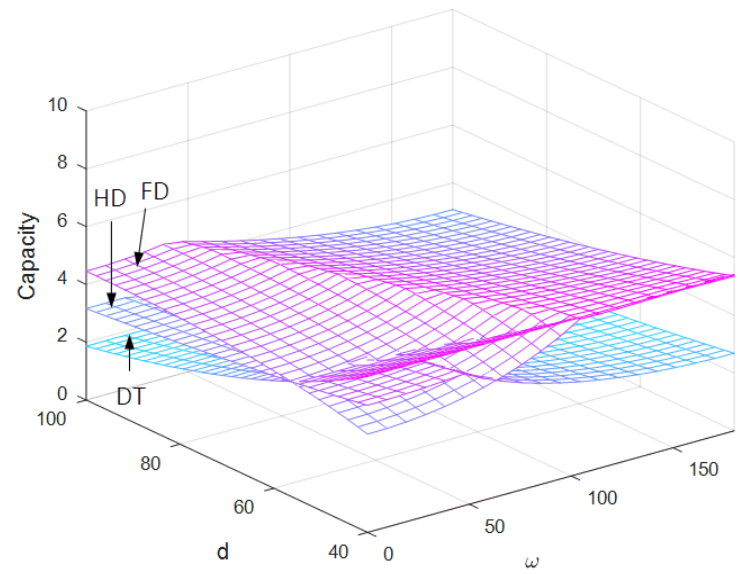

Fig. 7. Capacity with $P_{S}=0.1$ in independent-power-constraint mode.

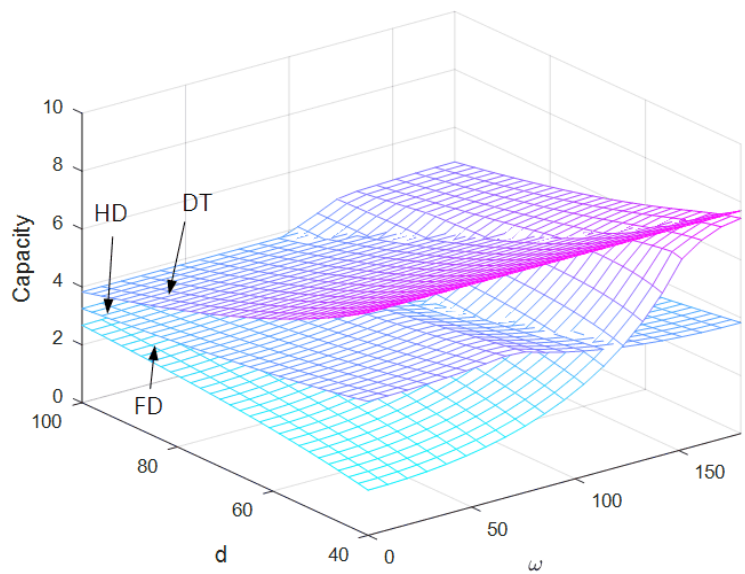

Fig. 8. Capacity with $P_{S}=0.5$ in independent-power-constraint mode.

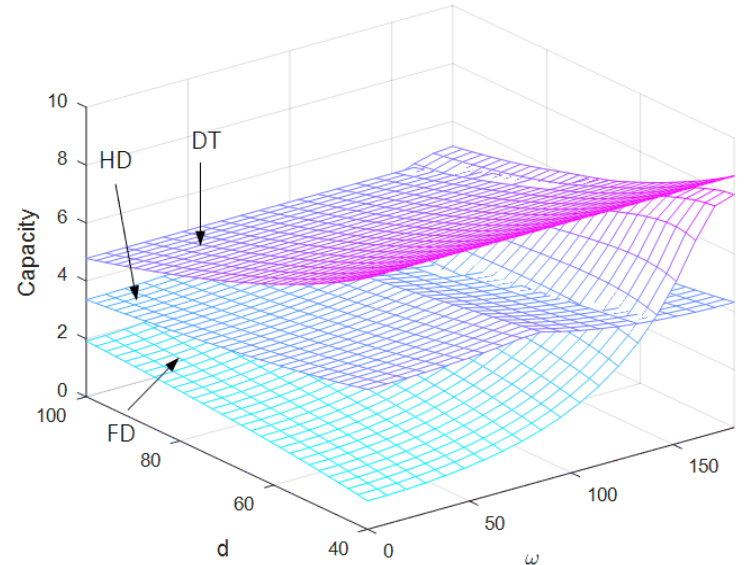

Fig. 9. Capacity with $P_{S}=1$ in independent-power-constraint mode. 
mode in $P_{S}=0.5$ is not significantly higher than that in total power constraint mode. When $P_{S}=1$, as shown in Fig. 9, although the capacity in HD mode grows a little, and the capacity of FD mode obviously becomes higher compared to Fig. 4., the DT capacity is still higher than that of the HD mode in most cases. Evidently, the system should prefer DT or FD mode when both $D$ and $\omega$ change and when the independent power constraint mode does not significant change the system optimal capacity mode selection.

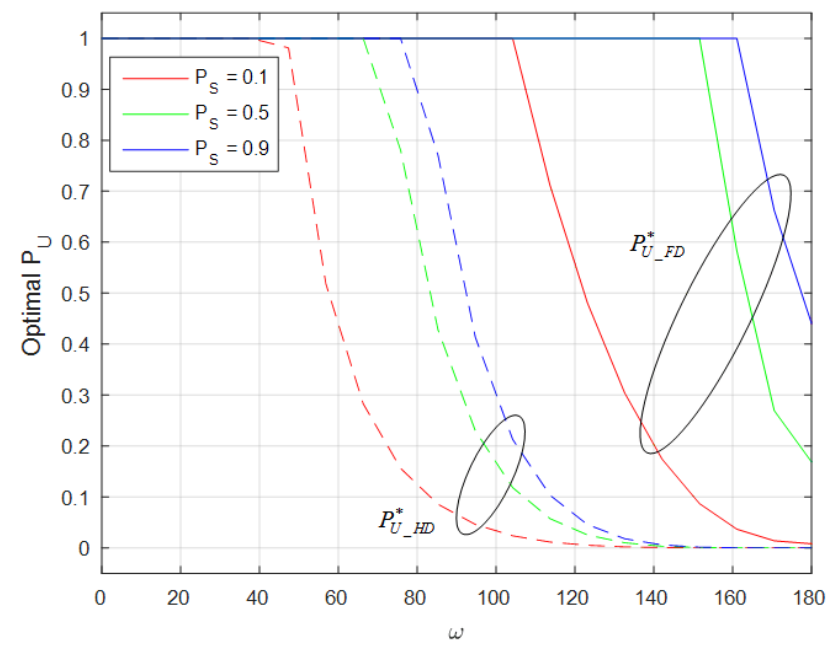

Fig. 10. Optimal $P_{U}$ under different $\omega$ in independent-power-constraint mode

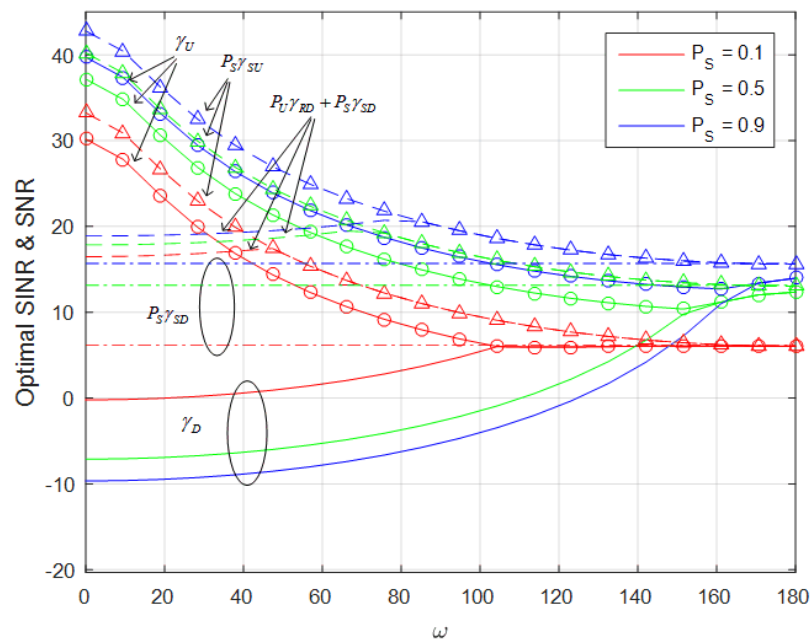

Fig. 11. Optimal SNRs and SINRs under different $\omega$ in independent-power-constraint mode.

2) For a more intuitive comparison, we are setting $P_{S}=0.1,0.5,0.9$ in both Fig. 10 and Fig. 11. In Fig. 10, comparing to Fig. 5, although the upper bound of the $P_{U}$ and $P_{S}$ have been improved, it still happens that $P_{U}^{*}$ has been limited for some $\omega$. And it increases sharply if $\mathrm{UAV}$ is far from $D$, indicating that the distance between $\mathrm{UAV}$ and $D$ has a strong impact on the optimal capacity. In Fig. 11, compare with Fig. 6, the values of upper curves $\gamma_{U}$ and $P_{S} \gamma_{S U}$ are rarely changed, while the values of $\gamma_{D}$ and $P_{U} \gamma_{R D}+P_{S} \gamma_{S D}$ are shifting up much in 
$P_{S}=0.9$, indicating that the increasing upper bound of $P_{U}$ makes more influence when $P_{S}$ is large, while $\gamma_{D}$ and $P_{U} \gamma_{R D}+P_{S} \gamma_{S D}$ are still mainly influenced by $P_{S}$, in which case it is relative small influenced by the upper bound of $P_{U}$. When $P_{U}$ upper bound increases, $\gamma_{U}$ and $P_{S} \gamma_{S U}$ increase accordingly, which also speeds up $\gamma_{D}$ and $P_{U} \gamma_{R D}+P_{S} \gamma_{S D}$ to convergence with $\gamma_{U}$ and $P_{S} \gamma_{S U}$ to improve the system capacity, as shown in Fig. 9. But in any case, if the system has the possibility of DT transmission, its performance is still better than that in both FD and HD modes.

\subsection{The Change of SI Threshold}

We then analyze the minimum value of the SI cancellation capability if the system does not need optimal power constraints operation from (18). In other words, sometimes SI is cancelled too much so that the power limitation operation is required. We may set $h=10, r=40, a=3$, $d=50$.

In total power constraint mode, as shown in Fig. 12, when UAV is more closer to $D$, the smaller threshold of SI cancellation can be obtained. When $P_{S}$ approaches 0.5 , there exists a

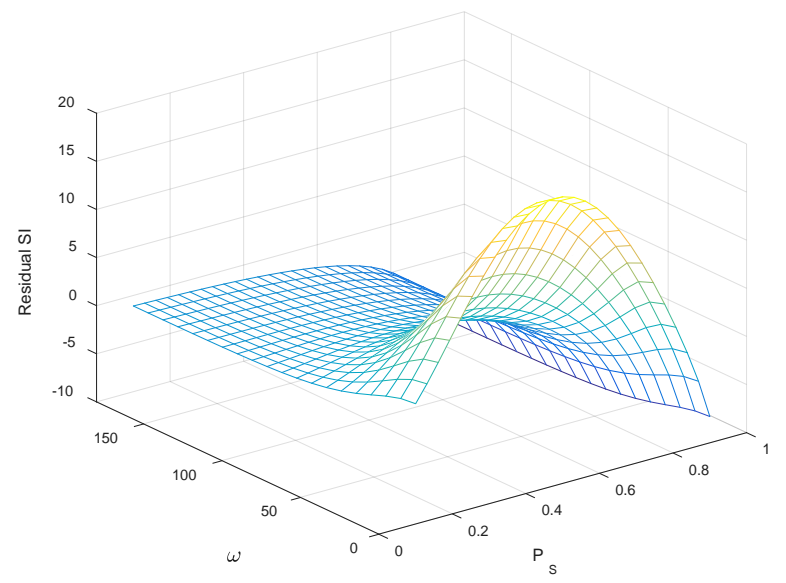

Fig. 12. The change of SI threshold in total power constraint.

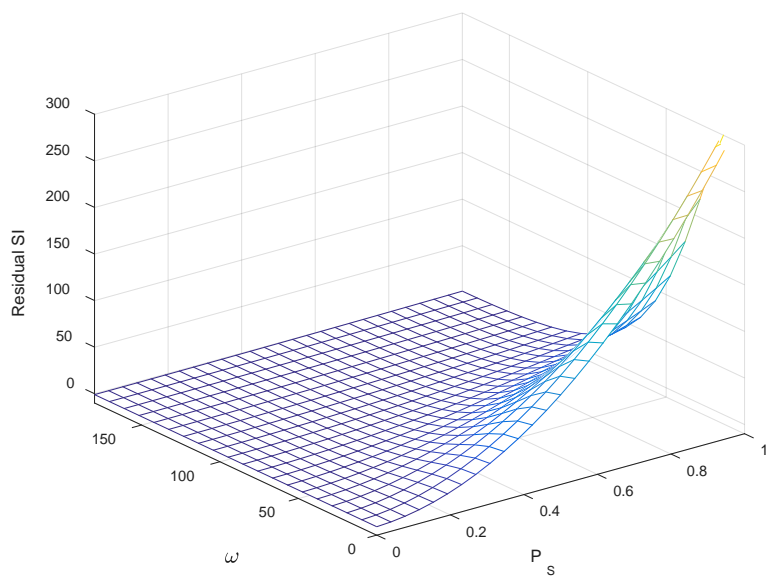

Fig. 13. The change of SI threshold in independent power constraint. 
highest point in the surface. But the entire curved surface does not take a value as the axial plane symmetry. The SI threshold in $P_{S}$ closed to 1 is obvious smaller than that in 0 . Meanwhile, it is necessary to improve the SI cancellation ability to achieve the optimal capacity when UAV keeps closed to $D$.

In independent power constraint mode, as shown in Fig. 13, compare to Fig. 12, when $P_{S}$ increases gradually and UAV is closed to $S$, the SI threshold is increased sharply so that the UAV almost does not need to cancel the SI, and the system does not need to conduct power constraint. Of course, this is just a result of the calculation and the actual situation may certainly be different. Furthermore, no matter how much the SI is being cancelled, it is still need to carry out power constraints operation.

\subsection{Transmit Power Adaption}

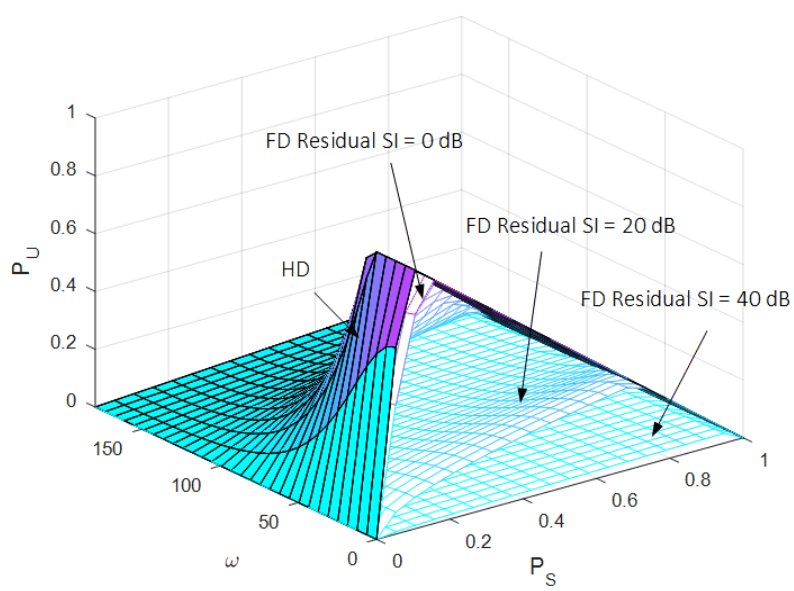

Fig. 14. The optimal $P_{U}$ in total power constraint.

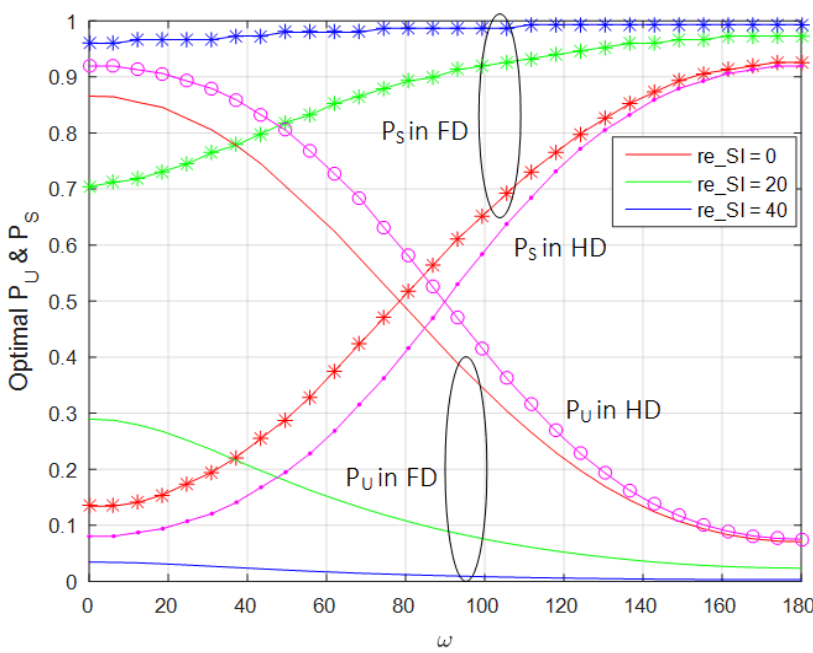

Fig. 15. The change of optimal $P_{S}$ under optimal $P_{S}$ in total power constraint.

In practical scenarios, there may exist no direct communication path between $S$ and $D$. Therefore, we discuss the hybrid mode selection without considering DT. 
We first analyze the changes of $P_{U}$, and then observe the change of $P_{S}$ under the optimal $P_{U}$. the simulation parameters are $K=(1 * 1 * 1 / 3) /(4 * p i)^{2}, h=10, r=40, a=3, d=40$.

In total power constraint mode, $P_{S}$ changes from $0-1$, according to (16) and (17), the optimal $P_{U}$ is shown in Fig. 14. We choose the case of residual $S I=0,20,40 d B$. As the HD transmission mode is not affected by SI, we may use a deep color to represent its $P_{U}$ and a light color to represent the there optimal $P_{U}$ under the change of SI. Even when the SI is complete cancelled, the $P_{U}$ in HD mode is still slightly higher than that in FD mode. However, due to the FD mode channel capacity is higher, it can be speculated that the capacity in FD will be higher, reflecting the benefits of the FD mode - with a smaller transmission power to achieve a larger capacity.

From Fig. 14, when UAV is getting closer to $D, P_{U}^{*}$ starts declining. When $\omega$ is closed to $180^{\circ}, P_{U}$ even almost equals to 0 , which is the same analysis results as Fig. 5. When $P_{S}$ changes from 0 to 1 . From (16) and (17), within the range of available power, $P_{U}$ is increased with the increases of $P_{S}$. When it exceed the available power, i.e. $P_{U} \geq 1-P_{S}, P_{U}$ will vary linearly with $P_{S}$, i.e. $P_{U}=1-P_{S}$. In other words, when $\omega$ is fixed, the curves will always first increase with the increase of $P_{S}$, and then go straight down. As all the $P_{U}^{*}$ of FD and HD will increase to up to $1-P_{S}$, we can notice that there are 4 overlapping surfaces in Fig. 14. And the larger residual SI, the slower growth of $P_{U}^{*}$ with the increase of $P_{S}$, and much later to achieve the highest point.

Fig. 15 shows the corresponding $P_{S}$ to the largest $P_{U}$ in $\omega$ changes, i.e. $P_{S}=\underset{P_{V} \leqslant P_{\text {max }}}{\arg \max } P_{U}$. As can be seen from this figure, with the increases of $\omega$, when UAV is closed to $D$, the actual optimal $P_{U}$ reduces gradually, while the corresponding $P_{S}$ increases gradually. When the SI is completely cancelled, $P_{U}$ reaches the maximum, the corresponding required $P_{S}$ reaches the least. When the residual SI is $40 \mathrm{~dB}$, the maximum $P_{U}$ is almost 0 , the corresponding $P_{S}$ will meet the maximum value, but it can also be predicted that the system capacity is very small. From the numerical values of the figure, almost all $P_{S}+P_{U}$ are equal to 1 , that is to say, almost all the optimal $P_{U}$ is taking the maximum available value. At the same time, we can also see that in HD mode, $P_{S}$ and $P_{U}$ are completely symmetrical.

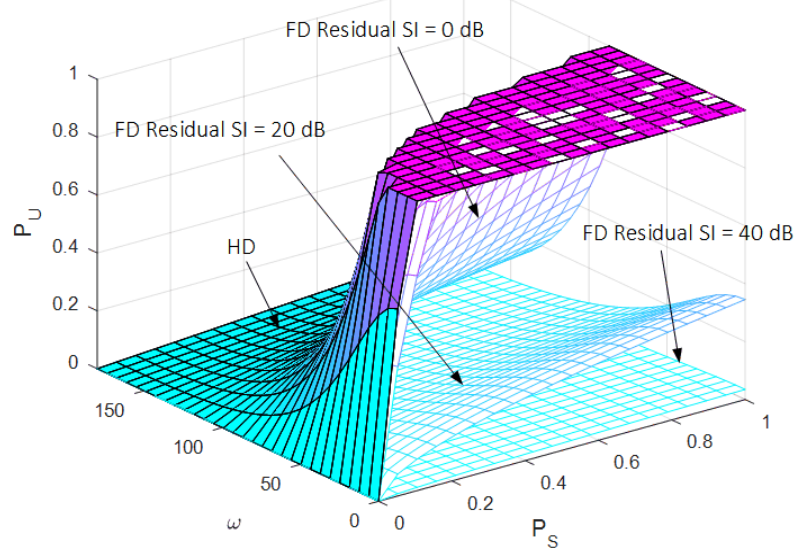

Fig. 16. The optimal $P_{U}$ in independent power constraint. 


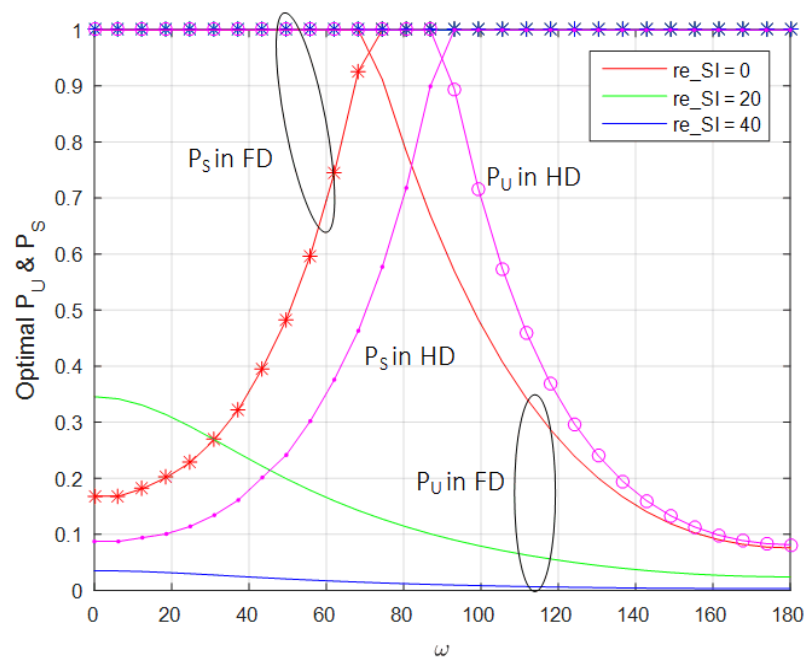

Fig. 17. The change of optimal $P_{S}$ under optimal $P_{S}$ in independent power constraint.

In independent power constraint mode, as shown in Fig. 16, when the residual SI is 0, the $P_{U}^{*}$ in FD mode is still higher than that in HD mode. In fact, compared to Fig. 14, the surface is growing to a flat top in $P_{U}=1$, corresponding to the upper limit of the available energy of $P_{U}$. Namely, the independent power constraint mode increased the available energy of $P_{U}$ under the conditions of $P_{U} \leq 1-P_{S}$.

Fig. 17 also shows the corresponding $P_{S}$ when $\omega$ changes in the case of largest $P_{U}$. When residual SI is $20 \mathrm{~dB}$ or $40 \mathrm{~dB}$, the curves of $P_{S}$ have overlapped, if the residual SI is larger, the largest $P_{S}$ and $P_{U}$ will be easy to reach the maximum. Compare with Fig. 15, the higher upper limit of $P_{U}$ and $P_{S}$, the more likely for this parameter to get closer to the upper limit.

\subsection{Maximum Capacity}

From the above analysis, for a given SI cancellation ability, with the change of $D, \omega$ and $P_{S}$, the capacity in FD and HD modes may also change.

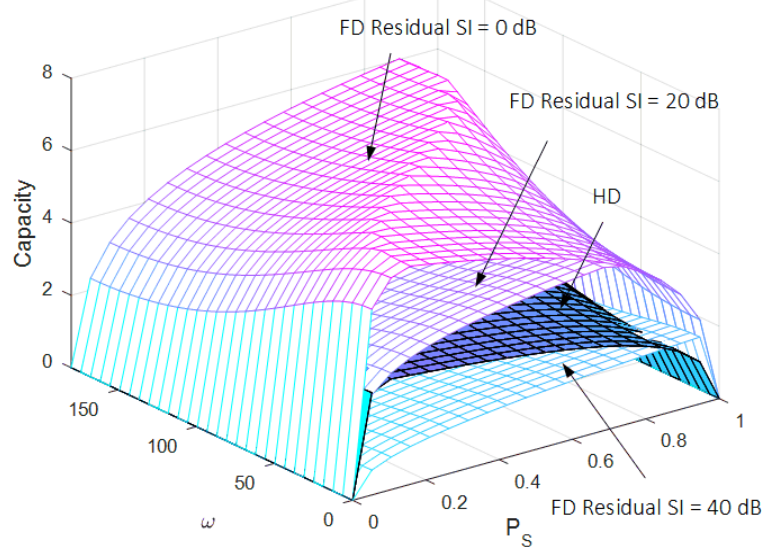

Fig. 18. The capacity changes in total power constraint. 


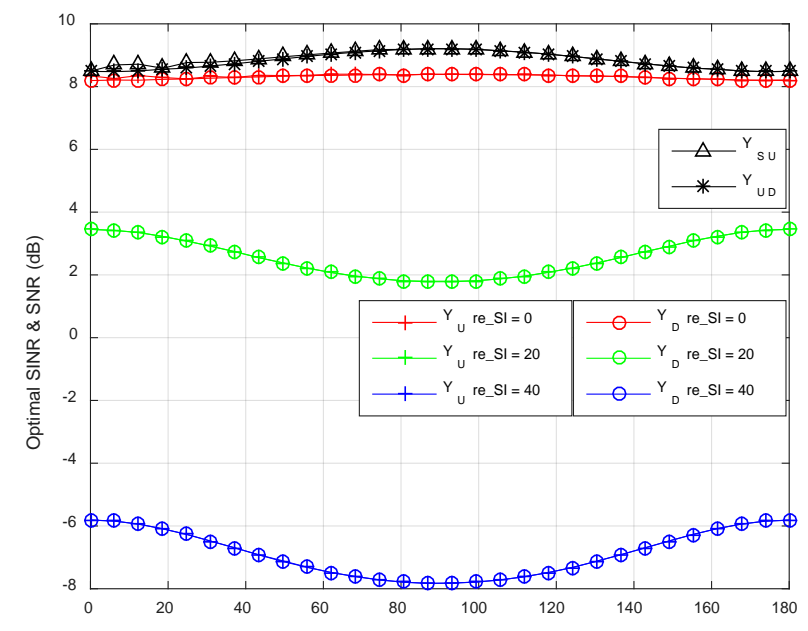

Fig. 19. The capacity changes in total power constraint

1) Max Capacity Under Different Situation: Let us first analyze the capacity with the change of $P_{S}$ and $\omega$ under different residual SI, followed by analyzing them under the optimal capacity condition. In this case, we make $K=(1 * 1 * 1 / 3) /(4 * \pi)^{2}, h=10, r=40, d=50$, $a=3$.

In total power constraint mode, as shown in Fig. 18, when $P_{S}=0$ or $P_{S}=1$, the surfaces are sharply reduced to 0 because the source or UAV has no power. When the residual SI is $40 \mathrm{~dB}$, the capacity in FD mode will be less than that in HD mode, indicating that selecting the HD mode will be better if the SI cancellation ability is poor. When the residual SI is $20 \mathrm{~dB}$, the capacity in FD mode becomes better than in HD mode. In order to make the figure clear, we do not draw another curved surfaces of $20-40 d B$ residual SI. It can be known that the curved surfaces must be undulated and overlapped with the HD mode curved surface, making some points be higher while some points are lower.

Meanwhile, the ups and downs of the surface are affected by the change of $P_{S}$ and $\omega$, making us draw the curves of $P_{S}$ and $P_{U}$ which corresponding to the maximum capacity in Fig. 18, as shown in Fig. 19. The SINRs in UAV and $D$ in optimal capacity are always equal to each other in any SI cancellation case, implying that in maximum capacity, the SINRs and SNR in UAV and $D$ are always identical. Although the SNR in HD mode is higher than SINRs in FD, it may not be play a decisive role in system maximum capacity. When $\omega=90^{\circ}$, the curves of HD mode and $S I=0$ reach the highest point, while the others reaches its minimum point. The residual SI affects the trend of SNRs and SINRs.

In independent power constraint mode, Fig. 20 has shown the capacity under the change of $P_{S}$ and $\omega$. When $P_{S}=0, S$ dose not has transmit power, and the curved surface is 0 . The change of capacity is similar to Fig. 18 in $P_{S}=0-0.5$ and the capacity is slightly higher, because $P_{U} \leq 1$. Meanwhile, $P_{U}$ and $P_{S}$ are not mutual restraint, so the surface does not tend to 0 while $P_{S}=1$.

In Fig. 21, it shows the change of $P_{U}$ and $P_{S}$ under the maximum capacity. In this part, we draw the curves of SINRs and SNRs diversification. Compare to Fig. 19, the value is slightly higher, and the curves in HD mode and $S I=0$ in FD mode in $\omega \in\left(0^{\circ}-70^{\circ}\right)$ are very closed 
to each other, because in FD mode, it has assumed there is no direct transmission and the SI is completely cancelled.

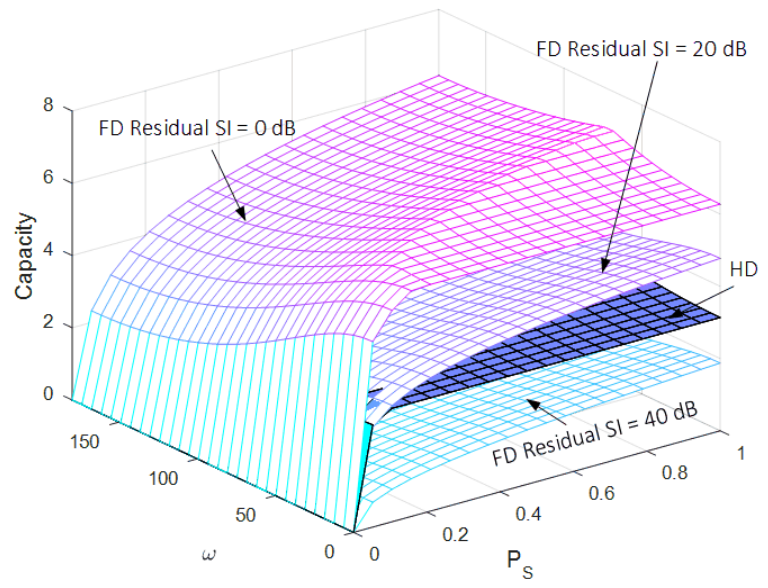

Fig. 20. The capacity changes in independent power constraint.

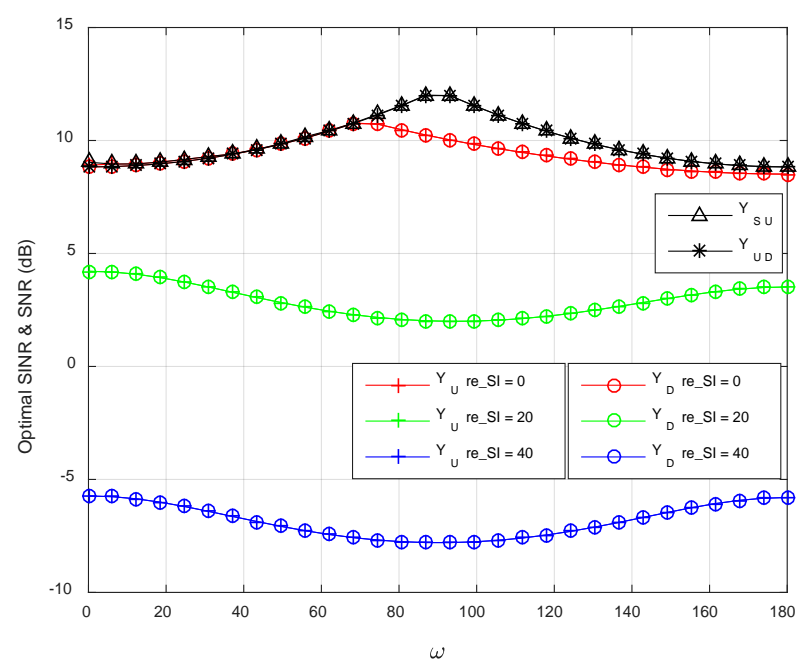

Fig. 21. The capacity changes in independent power constraint

2) The Comparison of Capacity Under Different SI Cancellation: From Fig. 18 and Fig. 20, in independent power constraint mode and total power constraint mode, the optimal capacity changes are no less than 2 times from the above analysis. In order to achieve the best choice between energy usage and maximum capacity, we compare the two cases of capacity, as shown in Fig. 22. Given the same SI cancellation ability, the maximum capacities difference between the two energy allocation schemes are small, and the weaker SI cancellation ability leads to the less maximum capacity. This shows that increasing the available energy of $P_{S}$ and $P_{U}$ cannot significantly improve the system's capacity. When the residual SI is only $30 \mathrm{~dB}$, the capacity in HD mode is better than FD mode, and we should give a higher priority to choose $\mathrm{HD}$ mode at this time. When the residual SI is between $20 \mathrm{~dB}$ to $30 \mathrm{~dB}$, with the change of UAV flight position, the capacity in FD mode will be lower than HD mode in the vicinity of $90^{\circ}$, and we can use the hybrid mode selection strategy to improve the system overall capacity. The weaker the SI cancellation ability, the weaker the impact of energy allocation strategy on FD 
mode, so we can choose total power constraint mode to save energy in these cases.

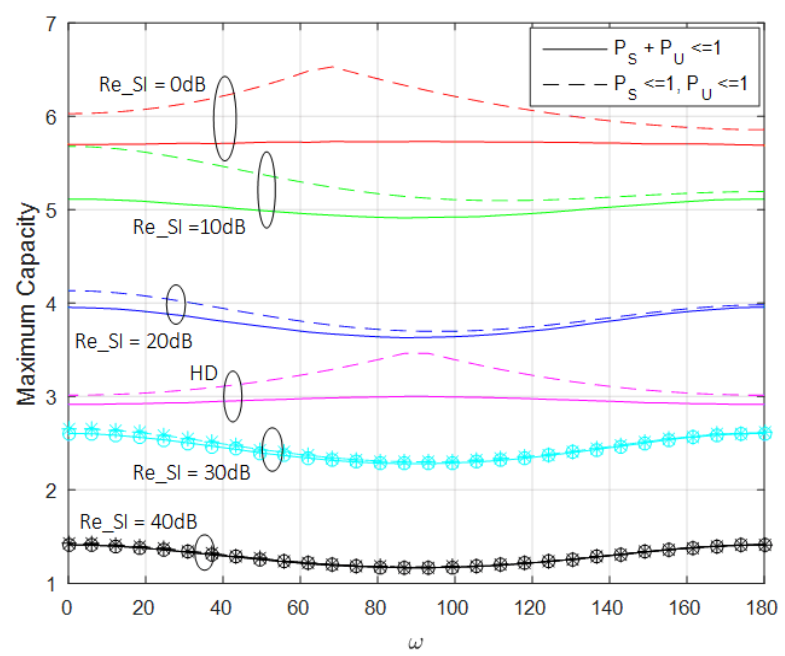

Fig. 22. The maximum capacity comparison.

\section{Conclusion}

We study the hybrid UAV capacity and power allocation scheme in this paper. At first, if the system has the chance to conduct DT mode, it should be preferred in most cases. When there exists no DT mode in the system and the SI cancellation ability is poor, we need to adopt a hybrid transmission strategy to achieve the optimal capacity. At the same time, the impacts of two power allocation schemes on capacity are no distinctive most of the time, implying it should give priority to the first power allocation scheme for optimizing the capacity. Furthermore, there still exist many contents which worthy of further investigation. Our current work only focuses on the capacity and power allocation, while the other studies such as energy harvesting and effective capacity will be further investigated. In addition the studies of enlarged scenarios of multiple UAVs and multiple nodes are more closed to the practical scenarios and can be further studied.

\section{References}

[1] F. Ono, H. Ochiai, and R. Miura, “A wireless relay network based on unmanned aircraft system with rate optimization,” IEEE Transactions on Wireless Communications, vol. 15, no. 11, pp. 7699-7708, 2016. Article (CrossRef Link)

[2] Z. Zhang, K. Long, J. Wang, and F. Dressler, "On swarm intelligence inspired self-organized networking: its bionic mechanisms, designing principles and optimization approaches,” IEEE Communications Surveys \& Tutorials, vol. 16, no. 1, pp. 513-537, 2014. Article (CrossRef Link)

[3] Z. Zhang, K. Long, and J. Wang, "Self-organization paradigms and optimization approaches for cognitive radio technologies: a survey,” IEEE Wireless Communications, vol. 20, no. 2, pp. 36-42, 2013. Article (CrossRef Link)

[4] O. S. Oubbati, A. Lakas, N. Lagraa, and M. B. Yagoubi, "UVAR: An intersection UAV-assisted VANET routing protocol," in Proc. of IEEE Wireless Communications and Networking Conference (WCNC), pp. 1-6, 2016. Article (CrossRef Link) 
[5] D. Orfanus, E. D. Freitas, and F. Eliassen, "Self-organization as a supporting paradigm for military UAV relay networks,” IEEE Communications Letters, vol. 20, no. 4, pp. 804-807, 2016. Article (CrossRef Link)

[6] K. Li, W. Ni, X. Wang, and R. P. Liu, "EPLA: Energy-balancing packets scheduling for airborne relaying networks," in Proc. of IEEE International Conference on Communications (ICC), pp. 6246-6251, 2015. Article (CrossRef Link)

[7] Z. M. Fadlullah, D. Takaishi, H. Nishiyama, N. Kato, and R. Miura, "A dynamic trajectory control algorithm for improving the communication throughput and delay in UAV-aided networks," IEEE Network, vol. 30, no. 1, pp. 100-105, 2016. Article (CrossRef Link)

[8] F. Ahdi and S. Subramaniam, "Using unmanned aerial vehicles as relays in wireless balloon networks," in Proc. of IEEE International Conference on Communications (ICC), pp. 3795-3800, 2015. Article (CrossRef Link)

[9] T. J. Willink, C. C. Squires, G. W. K. Colman, and M. T. Muccio, "Measurement and characterization of low-altitude air-to-ground MIMO channels," IEEE Transactions on Vehicular Technology, vol. 65, no. 4, pp. 2637-2648, 2016. Article (CrossRef Link)

[10] Y. Zeng, R. Zhang, and T. J. Lim, "Throughput maximization for UAV-enabled mobile relaying systems," IEEE Transactions on Communications, vol. 64, no. 12, pp. 4983-4996, 2016. Article (CrossRef Link)

[11] A. Chamseddine, O. Akhrif, G. Charland-Arcand, F. Gagnon, and D. Couillard, "Communication relay for multi-ground units with unmanned aerial vehicle using only signal strength and angle of arrival," IEEE Transactions on Control Systems Technology, vol. 25, no. 1, pp. 286-293, 2017. Article (CrossRef Link)

[12] Y. Zhou, N. Cheng, N. Lu, and X. S. Shen, "Multi-UAV-Aided Networks: Aerial-ground cooperative vehicular networking architecture," IEEE Transactions on Vehicular Technology, vol. 10, no. 4, pp. 36-44, 2015. Article (CrossRef Link)

[13] M. Horiuchi, H. Nishiyama, N. Kato, F. Ono, and R. Miura, "Throughput maximization for long-distance real-time data transmission over multiple UAVs," in Proc. of IEEE International Conference on Communications (ICC), pp. 1-6, 2016. Article (CrossRef Link)

[14] M. A. Araghizadeh, P. Teymoori, N. Yazdani, and S. Safari, "An efficient medium access control protocol for WSN-UAV,” Ad Hoc Networks, vol. 52, pp. 146-159, 2016. Article (CrossRef Link)

[15] Z. Zhang, K. Long, A. V. Vasilakos, and L. Hanzo, "Full-duplex wireless communications: challenges, solutions and future research directions," IEEE Processings, vol. 104, no. 7, pp. 1369-1409, 2016. Article (CrossRef Link)

[16] Z. Zhang, X. Chai, K. Long, A. V. Vasilakos, and L. Hanzo, "Full duplex techniques for 5G networks: self-interference cancellation, protocol design, and relay selection," IEEE Communication Magazine, vol. 53, no. 5, pp. 128-137, 2015. Article (CrossRef Link)

[17] M. Duarte, A. Sabharwal, V. Aggarwal, R. Jana, K. Ramakrishnan, C. W. Rice, and N. Shankaranarayanan, "Design and characterization of a full-duplex multiantenna system for WiFi networks," IEEE Transactions on Vehicular Technology, vol. 63, no. 3, pp. 1160-1177, 2014. Article (CrossRef Link)

[18] T. Riihonen, S. Werner, and R. Wichman, "Hybrid full-duplex/half-duplex relaying with transmit power adaptation," IEEE Transactions on Wireless Communications, vol. 10, no. 9, pp. 3074-3085, 2011. Article (CrossRef Link)

[19] B. Zhong and Z. Zhang, "Opportunistic two-way full-duplex relay selection in underlay cognitive networks," IEEE Systems Journal, vol. 12, no. 1, pp. 725-734, 2016. Article (CrossRef Link)

[20] H. Cui, M. Ma, L. Song, and B. Jiao, "Relay selection for two-way full duplex relay networks with amplify-and-forward protocol," IEEE Transactions on Wireless Communications, vol. 13, no. 7, pp. 3768-3777, 2014. Article (CrossRef Link)

[21] C. Liu, M. Ma, and B. Jiao, "A hybrid decode-and-forward relaying scheme for full duplex wireless relay networks," in Proc. of IEEE 83th Vehicular Technology Conference Spring (VTC-S), pp. 1-5, 2016. Article (CrossRef Link)

[22] Y. Li, T. Wang, Z. Zhao, and M. Peng, "Relay mode selection and power allocation for hybrid one-way/two-way half duplex/full-duplex relaying,” IEEE Communications Letters, vol. 19, no. 7, 
pp. 1217-1220, 2015. Article (CrossRef Link)

[23] S. Luo, P. Liu, and S. Panwar, "Full-duplex relaying in an infrastructure-based wireless network," in Proc. of IEEE 80th Vehicular Technology Conference Fall (VTC-F), pp. 1-6, 2014. Article (CrossRef Link)

[24] L. Han and J. Mu, "Hybrid half-duplex/full-duplex multi-hop relaying schemes: outage performance and power optimization,” Ad Hoc Networks, vol. 58, pp. 54-61, 2017. Article (CrossRef Link)

[25] D. Korpi, T. Riihonen, K. Haneda, K. Yamamoto, and M. Valkama, "Achievable transmission rates and self-interference channel estimation in hybrid full-duplex/half-duplex MIMO relaying," in Proc. of IEEE 82th Vehicular Technology Conference Fall (VTC-F), pp. 1-5, 2015. Article (CrossRef Link)

[26] J. Lee and T. Q. S. Quek, "Hybrid full-/half-duplex system analysis in heterogeneous wireless networks,” IEEE Transactions on Wireless Communications, vol. 14, no. 5, pp. 2883-2895, 2015. Article (CrossRef Link)

[27] M. Feng, S. Mao, and T. Jiang, "Joint duplex mode selection, channel allocation, and power control for full-duplex cognitive femtocell networks," Digital Communications and Networks, vol. 1, no. 1, pp. 30-44, 2015. Article (CrossRef Link)

[28] Z. Lin, Y. Cai, W. Yang, and X. Xu, "Opportunistic relaying and jamming with robust design in hybrid full/half-duplex relay system," EURASIP Journal on Wireless Communications and Networking, vol. 2016, pp. 1-10, 2016. Article (CrossRef Link)

[29] A. E. Shafie, A. Sultan, and N. Al-Dhahir, "Physical-layer security of a buffer-aided full-duplex relaying system,” IEEE Communications Letters, vol. 20, no. 9, pp. 1856-1859, 2016. Article (CrossRef Link)

[30] G. Chen, P. Xiao, J. R. Kelly, B. Li, and R. Tafazolli, "Full-duplex wireless-powered relay in two way cooperative networks,” IEEE Access, vol. 5, pp. 1548-1558, 2017. Article (CrossRef Link)

[31] J. N. Laneman, D. N. Tse, and G. W. Wornell, "Cooperative diversity in wireless networks: efficient protocols and outage behavior," IEEE Transactions on Information Theory, vol. 50, no. 12, pp. 3062-3080, 2004. Article (CrossRef Link) 


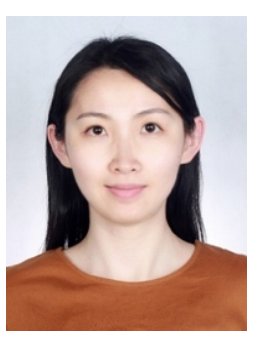

Qian Zeng, received her B.S. degree in Guangxi Normal University, Guangxi, China in 2008, the M.S. degree in Guilin University of Electronic Technology, Guilin, China in 2015. She is currently working toward the D.S. degree in University of Science and Technology Beiing (USTB), Beiing, China. Her current research interests include Unmanned Aerial Vehicles (UAVs) aided communication system, D2D communication system and cooperative communications. Email: zengqian617@foxmail.com.

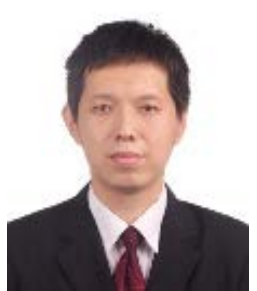

Wei Huangfu, received his M.S. and PhD. degrees in electronic engineering from Tsinghua University, Beijing, China in 1998 and 2001, respectively. He is currently an Associate Professor of the School of Computer and Communication Engineering in the University of Science and Technology Beijing (USTB). His main research interests include wireless communication networks, Internet of Things and big-data processing. Email: huangfuwei@ustb.edu.cn

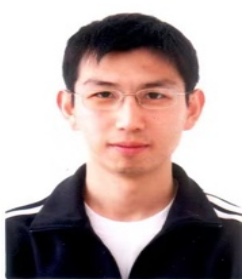

Tong Liu, received his B.E. degree in automatic control from Harbin Engineering University (HEU) in 2000, M.S. degrees in navigation guidance and control from Harbin Engineering University (HEU) in 2002 , and his Ph.D. degree in electrical engineering in 2006 from Harbin Institute of Technology (HIT) . From February 2006 to August 2006 he joined Huawei Technologies Ltd as an associate researcher. He is currently an associate professor with the School of information and communication engineering at Harbin Engineering University (HEU). His main research interests include tactical networking, software defined radio. Email: liutong@hrbeu.edu.cn. 